Dhaka Univ. J. Biol. Sci. 28(1): 21-35, 2019 (January)

\title{
MYCOFLORA ASSOCIATED WITH BIPOLARIS LEAF BLIGHT OF DIFFERENT WHEAT VARIETIES IN BANGLADESH
}

\author{
Mst Selina Momtaz ${ }^{1}$, Shamim Shamsi* and Tapan Kumar Dey ${ }^{2}$ \\ Department of Botany, University of Dhaka, Dhaka-1000, Bangladesh
}

Key words: Bipolaris leaf blight, Mycoflora, Wheat varieties

\begin{abstract}
Thirty five fungal species, representing 20 genera were found to be associated with Bipolaris leaf blight infected leaves of 21 wheat varieties, collected from eight districts (Dhaka, Gazipur, Dinajpur, Joypurhat, Pabna, Sirajgonj, Kushtia and Chuadanga) of Bangladesh. Fungi were isolated following tissue planting method. The isolated fungi were Alternaria alternata, A. triticina, Arthirinium sp., Aspergillus flavus, A. fumigatus, A. niger, A. terreus, Aspergillus sp., Bipolaris cynodontis, B. oryzae, B. sorokiniana, B. tetramera, B. victoriae, Bispora antenata, Chaetomium globosum, Chaetophoma sp., Cladosporium cladosporioides, Coniothyrium sp., Curvularia affinis, C. lunata, C. pallescens, Drechslera dematioidea, D. hawaiiensis, Epicoccum purpurascens, Eurotium sp., Fusarium moniliforme, F. nivale, $F$. semitectum, Nigrospora oryzae, N. sacchari, Penicillium digitatum, Pestalotiopsis guepinii, Rhizopus stolonifer, Syncephalastrum racemosum and Trichoderma viride. Bispora antenata is new record for Bangladesh.
\end{abstract}

\section{Introduction}

Wheat (Triticum aestivum L.) is the second most important cereal crop next to rice in Bangladesh. During 2016-17, total wheat production was 1.335 million tons from 0.435 million hectares of land. The country needs 5.5 million tons wheat, $80 \%$ of which are imported every year ${ }^{(1)}$. Still the yield level is much lower than the potential yield. Diseases play an important role in lowering yield in the country. Wheat is attacked by at least 20 different diseases in Bangladesh ${ }^{(2,3)}$, of which five are considered as major diseases, they are Bipolaris leaf blight (Bipolaris sorokiniana), leaf rust (Puccinia recondita), seedling blight (Bipolaris sorokiniana), foot and root rot (Sclerotium rolfsii Tode) and black point [B. sorokiniana, Alternaria alternata (Fr) Keissler, Curvularia lunata (Wakker) Boedijn and species of Fusarium Link]. Sadat and Choi reported wheat blast caused by Pyricularia graminis-tritici and P. oryzae from Bangladesh ${ }^{(4)}$.

*Author for correspondence: <prof.shamsi@gmail.com>. ${ }^{1}$ Department of Botany, Jagannath University, Dhaka-1100, Bangladesh. 2Senior Program Specialist (Crops) KGF, BARC Complex, Farmgate, Dhaka, Bangladesh. A part of Ph.D. Thesis of first author (MSM). 
Bipolaris sorokiniana (Sacc.) Shoemaker, teleomorph, Cochliobolus sativus (Ito and Kurib.) Drechsler ex Dastur, is the most important biotic constraint affecting wheat production in Bangladesh ${ }^{(5)}$. This fungus acts as a causal agent for various diseases like head blight, seedling blight, foliar blight/spot blotch, common root rot and black point of wheat, barley, other small cereal grains and grasses ${ }^{(6,7)}$. On an average, a South-Asian country loses $20 \%$ of crop yield through leaf blight ${ }^{(8)}$. Yield loss was estimated to be $15 \%$ in Bangladesh ${ }^{(9)}, 18-22 \%$ in India ${ }^{(10)}$. The study was conducted to find out the existence of mycoflora living on BpLB infected wheat phylloplane.

\section{Materials and Methods}

BpLB infected leaves in different varieties of wheat were collected from eight districts (Dhaka, Gazipur, Dinajpur, Joypurhat, Pabna, Sirajgonj, Kushtia and Chuadanga) of Bangladesh. Infected leaf samples were collected at grain filling stage and placed in clean brown paper bag, labeled properly and preserved at $4^{\circ} \mathrm{C}$ in refrigerator for future studies.

The fungi were isolated from the samples following tissue planting method on PDA (Potato Dextrose Agar) medium ${ }^{(11)}$. For surface sterilization first the diseased leaf samples were washed under running tap water and cut into $2^{2} \mathrm{~mm}$ pieces. Then cut pieces were sterilized in 10\% Chlorox for $2 \mathrm{~min}$ and then washed in sterilized water thrice. Finally, the inocula were placed inside the folds of a sterile blotting paper to remove excess surface water. After blot-drying, inocula were transferred to medium under aseptic conditions using laminar air flow. The inocula were placed in Petri plates containing sterilized potato dextrose agar (PDA) medium, each Petri plate contained $15 \mathrm{ml}$ of PDA medium with an additional of 1 drop (ca. $0.03 \mathrm{ml}$ ) of lactic acid which was used for checking the bacterial growth. A total number of 50 inocula were transferred in 10 Petri plates for each sample. Then the inoculated plates were incubated at room temperature $\left(25 \pm 2^{\circ} \mathrm{C}\right)$ for seven days. Fungi grew from the diseased leaves were transferred to separate PDA plates and PDA slants for further studies and preservation.

Detailed morphological studies of the fungal isolates were made in order to determine their identification. The microscopic structural characters of the isolated fungi were recorded under Nikon D 5000 digital camera. Then isolated fungi were identified based on following standard literatures ${ }^{(12-18)}$. All specimens, included in the present study were preserved in Mycology and Plant Pathology Laboratory, Department of Botany, University of Dhaka, Bangladesh.

\section{Results and Discussion}

Thirty five fungal species, representing 20 genera were found to be associated with BpLB infected leaves of 21 wheat varieties, collected from eight districts of Bangladesh. The isolated fungi were Alternaria alternata Keissler, A. triticina Prasada \& Prabhu, Arthrinium Kunze ex Fr., Aspergillus flavus Link, A. fumigatus Fresen., A. niger Tiegh., A. 
terreus Thom., Aspergillus sp. Link, Bipolaris cynodontis (Marig.) Shoem., B. oryzae (Breda De Haan) Shoem., B. sorokiniana (Sacc.) Shoem., B. tetramera (Mckinney) Shoem., B. victoriae (Meehan \& Murphy) Shoem., Bispora antenata (Pers.) Mason, Cheatomium globosum Kunze ex Fr., Chaetophoma Cooke, Cladosporium cladosporioides (Fresen.) de Vries, Coniothyrium sp. Corda, Curvularia affinis Boedijn, C. lunata Boedijn, C. pallescens Boedijn, Drechslera dematioidea (Bub. \& Wrob.) Subram. \& Jain, D. hawaiiensis (Bugnicourt) ex M.B. Ellis; Subram. \& Jain, Epicoccum purpurascens Ehrneb. ex Schlecht, Eurotium sp., Fusarium moniliforme Sheldon, F. nivale Ces., F. semitectum Berk. \& Rav., Nigrospora oryzae (Berk. \& Br.) Petch., N. sacchari (Speg.) Mason, Penicillium digitatum (Fr.) Sacc., Pestalotiopsis guepinii (Desm.) Stay., Rhizopus stolonifer (Ehrenb.: Fr.) Vuill, Syncephalastrum racemosum Cohn ex J. Schröt. and Trichoderma viride Pers.

\section{Taxonomic enumeration of Fungi}

Alternaria alternata (Fr.) Keissler 1912. Beih. Bot. Zbl. 29: 433

(Fig. 1A)

Colony usually black or olivaceous black, sometimes grey. Conidiophores golden brown, smooth, up to $50 \times 3-6 \mu \mathrm{m}$. Conidia formed in long, often branched chains, obclavate, obpyriform, ovoid or ellipsoidal, often with a short conidial or cylindrical beak, pale to mid golden brown, smooth or verruculose, with up to 8 transverse and usually several longitudinal or oblique septa, $21.6-52.4 \times 9.2-17.1$, beak pale, $2-5 \mu \mathrm{m}$ thick.

Specimen examined: BpLB infected leaves of wheat (Triticum aestivum L.), variety Kalyansona, vill.-WRC, dist. - Dinajpur, S Momtaz 116, 25 February 2011.

Alternaria triticina Prasada \& Prabhu 1963. Indian Phytopath. 15: 292-293

(Fig. 1B)

Colony discrete or effuse, dark blackish brown to black. Conidiophores up to 30 $\times 3-6$ $\mu \mathrm{m}$, golden or olivaceous brown, occasionally branched. Conidia solitary, obclavate, rostrate, golden brown, smooth, $27.2-76.3 \times 10.2-25.4 \mu \mathrm{m}$, body usually with $4-7$ transverse and several longitudinal or oblique septa, beak shorter than or the same length as the body, cylindrical, 3 - $5 \mu \mathrm{m}$ thick.

Specimen examined: BpLB infected leaves of wheat (Triticum aestivum L.), varietyBalaka, vill.-WRC, dist. - Dinajpur, S Momtaz 124, 25 March 2011.

Arthirinium Kunze ex Fr. 1821. Kunze in Kunze \& Schmidt.

(Fig. 1C)

Colonies effused, blackish brown. Conidia solitary, circular to subcircular, lateral and sometimes also terminal, frequently flattened and with a hyaline rim or germ slit, brown or dark brown, smooth, 0-septate, 9.3 - $10.2 \mu \mathrm{m}$.

Specimen examined: BpLB infected leaves of wheat (Triticum aestivum L.), varietyKanchan, vill.-BARI, dist. Gazipur, S Momtaz 111, 13 March 2011. 
Aspergillus flavus Link 1809. Magazin der Gesellschaft Naturforschenden Freunde Berlin 3(1): 16

(Fig. 1D)

Colonies effuse greenish. Mycelia well developed, septate, hyaline and profusely branched. Conidiophores $300-600 \mu \mathrm{m}$ long. Vasicles 10 - $35 \mu \mathrm{m}$ in diameter. Sterigmata $8-14 \times 3-5 \mu \mathrm{m}$. Conidia greenish, catenulate, globose or pyriform, smooth, $3-5 \mu \mathrm{m}$ in diameter.

Specimen examined: BpLB infected leaves of wheat (Triticum aestivum L.), varietySonalika, vill. - BARI, dist. - Gazipur, S Momtaz, 29, 3 April 2010.

Aspergillus fumigatus Fresenius 1863. Beitrage zur Mykologie 3: 81.

(Fig. 1E)

Colonies flat, olivaceous green. Conidiophores long, often with a foot cell, straight or flexuous, swollen at the apex into a spherical vesicle. Surface of vesicle covered by closely packed more or less clavate branches. Conidia catenulate, dry, usually globose, echinulate and smooth. Colonies of the fungus produced thousands of minute pale green conidia $(2-3 \mu \mathrm{m})$.

Specimen examined: BpLB infected leaves of wheat (Triticum aestivum L.), variety-Inia66, vill.- BARI, dist. - Gazipur, S Momtaz 22, 3 April 2010.

Aspergillus niger Van Tieghem 1867. Ann. Sci. Nat. Bot. Ser. 5, 8: 240.

(Fig. 1F)

Colonies effuse, black. Conidiophores brown $200-400 \times 7$ - $10 \mu \mathrm{m}$. Vesicles globose or sub globose, thick walled, commonly $20-50 \mu \mathrm{m}$, occasionally up to $100 \mu \mathrm{m}$ in diameter. Foot cell present. Sterigmata $20-30 \times 6-8 \mu \mathrm{m}$. Conidia dark brown, one celled, globose, 2 - 4 (5) $\mu \mathrm{m}$ in diameter.

Specimen examined: BpLB infected leaves of wheat (Triticum aestivum L.), varietyCiano-79, vill.-WRC, dist. - Dinajpur, S Momtaz 212, 23 March, 2011.

Aspergillus terreus Thom. 1918. Amer. J. Bot. 5(2): 85

(Fig. 1G)

Colonies pure brown or ochraceous brown, brighter at maturity. Reverse dull yellow. Conidiophores smooth, flexuous, commonly $100-265 \times 4-6 \mu \mathrm{m}$. Vasicles dome shaped, 9-17 $\mu \mathrm{m}$ in diameter. Sterigmata $4-7 \times 2-5 \mu \mathrm{m}$. Conidia pale brown, globose to slightly ellipsoidal, catenulate, 2 - $3 \mu \mathrm{m}$.

Specimen examined: BpLB infected leaves of wheat (Triticum aestivum L.), varietySonora, vill.- BARI, dist. - Gazipur, S Momtaz 49, 3 April 2010.

Aspergillus Link

(Fig. 1H)

Colonies flat, yellowish. Mycelium well-developed, septate, brown. Conidiophores very long, often with a foot cell, straight, $100-465 \times 5-6 \mu \mathrm{m}$, terminating in a globose head. Vasicles dome shaped, $8-16 \mu \mathrm{m}$. Surface of vesicle covered by closely packed more or less clavate sterigmata $5-8 \times 2-5 \mu \mathrm{m}$. Conidia hyaline, one celled, globose, echinulate, 3 - $5 \mu \mathrm{m}$. 

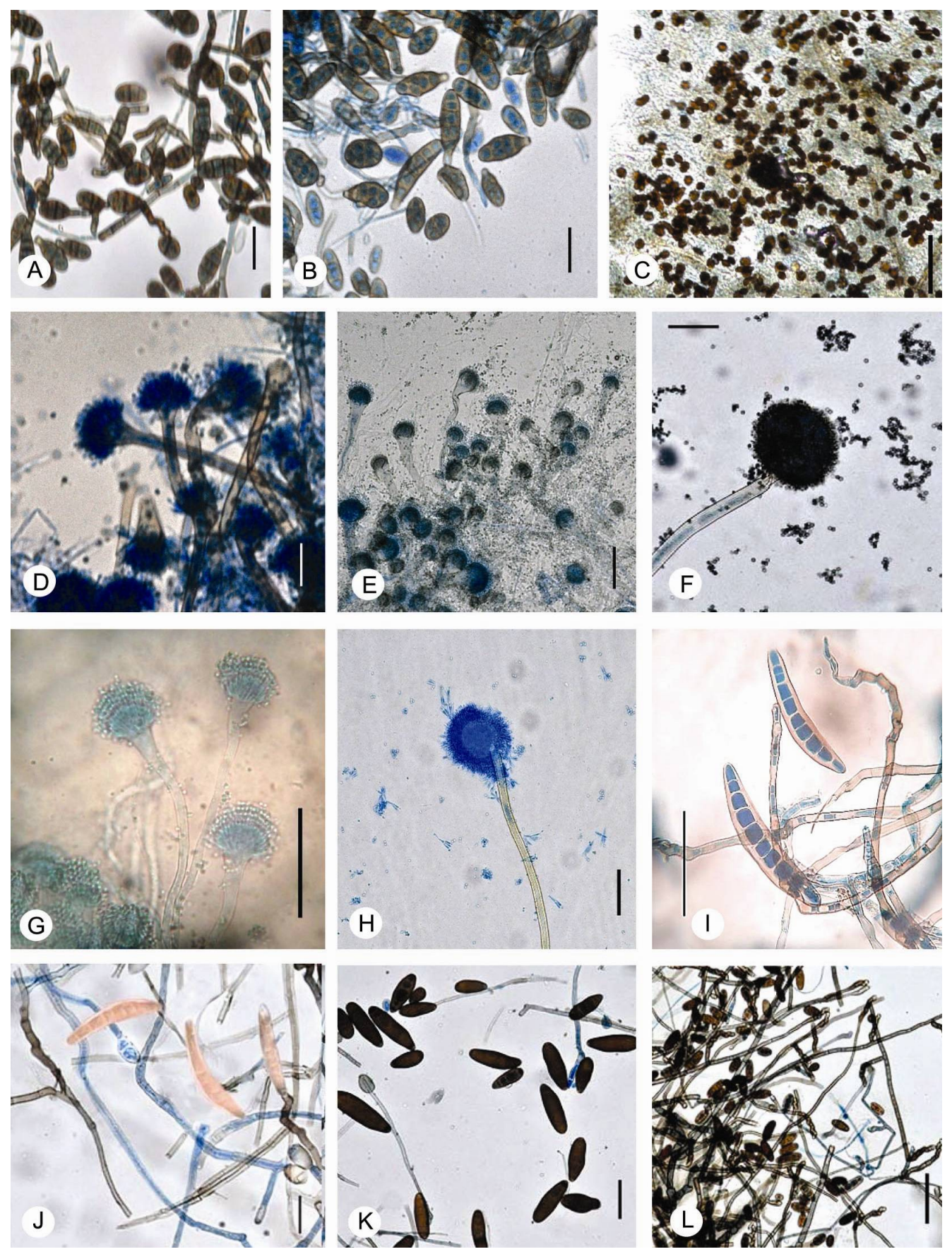

Fig. 1. Microphotograph showing conidiophores and conidia of A. Alternaria alternata, B. A. triticina, C. Arthinium sp., D. Aspergillus flavus, E. A. fumigatus, F. A. niger, G. A. terreus, H. Aspergillus sp., I. Bipolaris cynodontis; J.B. oryzae; K. B. sorokiniana and L. B. tetramera $($ Bar $=50 \mu \mathrm{m})$. 
Specimen examined: BpLB infected leaves of wheat (Triticum aestivum L.), varietyShatabdi, vill.- Farakpur, dist. -Kushtia, S Momtaz 296, 7 March, 2012.

Bipolaris cynodontis (Marignoni) Shoemaker 1959. Canadian J. Bot., 37(5): 883 (Fig. 1I)

Colonies on PDA medium dark ash to black, cottony, reverse black and mycelium profusely branched, septate, brown. Conidiophores pale to mid brown, arising singly or in groups of 2 or 3, short, straight or slightly bent. Conidia light to olivaceous brown, ellipsoid to ovate, broader in the middle with rounded ends, straight or slightly curved, uniform in colour, smooth, thin walled. 3 - 9 (usually 7 - 8) pseudoseptate, $35.6-59.5 \times 8.5$ $-13.6 \mu \mathrm{m}$.

Specimen examined: BpLB infected leaves of wheat (Triticum aestivum L.), varietySaurab, vill.- Vutiapara, dist.- Joypurhat, S Momtaz 425, 4 April 2013.

Bipolaris oryzae (Breda De Haan) Shoemaker 1959. Canadian J. Bot. 37(5): 883 (Fig. 1J)

Colony ash grey to dark greenish grey, mycelium fluffy, aerial, cottony. Conidiophores solitary or in small groups, straight or flexuous, pale to mid brown, Conidia olivaceous brown, curved, widest in the middle or just above middle, tapering to rounded ends, base more rounded, not flat or definite, 89.1 - $126.9 \times 18.9$ - 21.6 (17) $\mu \mathrm{m}, 8$ 13 pseudoseptate.

Specimen examined: BpLB infected leaves of wheat (Triticum aestivum L.), varietyShatabdi, vill.- BARI, dist. - Gazipur, S Momtaz 11, 10 March 2010.

Bipolaris sorokiniana (Sacc.) Shoemaker 1959. Canadian J. Bot. 37(5): 883

(Fig. 1K)

Colonies olivaceous brown to very dark becoming generally lighter towards the periphery, margin mostly smooth. Conidiophores brown, short, erect, in most cases single, bearing 1-6 conidia. Conidia ellipsoid, dark brown, mostly straight or slightly curved, wall thick but less so towards the ends, broadest in the middle, ends rounded, scar clear within the basal cell. Terminal portion of the end cells subhyaline, 6-9 pseudoseptate , $48.0-88.6 \times 17.2-25.8 \mu \mathrm{m}$.

Specimen examined: BpLB infected leaves of wheat (Triticum aestivum L.), varietyShatabdi, vill.- Doripara, dist. - Joypurhat, S Momtaz 362, 4 April 2013.

Bipolaris tetramera (Mckinney) Shoemaker 1959. Canadian J. Bot. 37(5): 883

(Fig. 1L)

Colonies on PDA brown to olivaceous brown, mat, reverse brown, remarkable colony margin with brown band also noticed. Conidiophores brownish, single or in clusters of 2 to 3, conidia almost in cluster. Brown, ellipsoid, mostly cylindric, straight with broadly rounded ends, lighter towards the terminal cells, 3 pseudoseptate, 22.6 $35.6 \times 10.2-12.8 \mu \mathrm{m}$.

Specimen examined: BpLB infected leaves of wheat (Triticum aestivum L.), varietyKanchan, vill.- BARI, dist.- Gazipur, S Momtaz 109, 12 February 2011. 
Bipolaris victoriae (Meehan \& Murphy) Shoemaker 1959. Canadian J. Bot. 37(5):883

(Fig. 2A)

Colony color and growth characters on PDA very similar to those of B. sorokiniana, except in this case the conidia little lighter in color, slender and slightly curved. Conidiophores solitary or in small groups, straight or flexuous, pale to mid brown. Conidia long, ellipsoid, straight or slightly curved, thin walled, pale or mid-pale golden brown, 8-10 pseudoseptate, 46.8 - $70.5 \times 12.6-18 \mu \mathrm{m}$.

Specimen examined: BpLB infected leaves of wheat (Triticum aestivum L.), varietyShatabdi, vill.- BARI, dist.- Gazipur, S Momtaz 531 , 10 April 2013.

Bispora antenata (Pers.) Mason 1953. Can. J. Bot. 31: 582

(Fig. 2B)

Colony effuse, black. Mycelium dark, conidiophores dark brown, short, simple or sparingly branched, $5-30 \times 2-5 \mu \mathrm{m}$. Conidia brown or dark brown, oblong, 2-celled or less often 3-celled, with thick black band at the septa, catenulate, produced acropetally, 14.1 - $17.8 \times 7.2-8.3 \mu \mathrm{m}$. Saprophytic on leaves, woods.

Specimen examined: BpLB infected leaves of wheat (Triticum aestivum L.), varietyAghrani, vill.-WRC, dist. - Dinajpur, S Momtaz 192, 24 March 2011.

The fungus is new record for Bangladesh.

Chaetomium globosum Kunze ex Fr. 1829. Systema Mycologicum 3: 255

(Fig. 2C)

Colonies punctiform. Perithecia dark brown to black and clothed, asci soon disappearing. The lemon shaped, brown ascospores free from their asci through the ostiole, $5.2-6 \times 2.8-4 \mu \mathrm{m}$.

Specimen examined: BpLB infected leaves of Wheat (Triticum aestivum L.), variety-Seri82, vill.-Sadar, dist. -Chuadanga, S Momtaz 318, 17 March 2013.

Chaetophoma Cooke 1878. Grevillea 7(41): 25

(Fig. 2D)

Colonies greish black, cottony. Reverse grayish black. Pycnidia dark, superficial, without ostiole, in dense or loose clusters, seated on a subiculum of interwoven hyphae, very small, globose to irregular, $52.2-66.6 \times 41.4-61.2 \mu \mathrm{m}$. Conidia ovate or elliptic, hyaline, 1-celled, very small 3 - $5 \mu \mathrm{m}$. Saprophytic on plant material.

Specimen examined: BpLB infected leaves of Wheat (Triticum aestivum L.), varietySaurav, vill.-Kazirhat, dist. -Pabna, S Momtaz 326, 5 April 2013.

Cladosporium cladosporioides (Fresen.) G.A. de Vries 1952. Contribution to the knowledge of the genus Cladosporium: 57

(Fig. 2E)

Colonies effuse olive green, or olivaceous brown, velvety. Conidiophores sometimes up to $350 \mu \mathrm{m}$ long, olivaceous brown, smooth or verruculose. Ramo-conidia 0 - 1 septate, up to $30 \mu \mathrm{m}$ long, $2-5 \mu \mathrm{m}$ thick, smooth. Conidia formed in long branched chains, pale olivaceous brown, mostly 0-septate, ellipsoidal or lemoniform, smooth, 4 - $10 \times 2$ - $4 \mu \mathrm{m}$. 

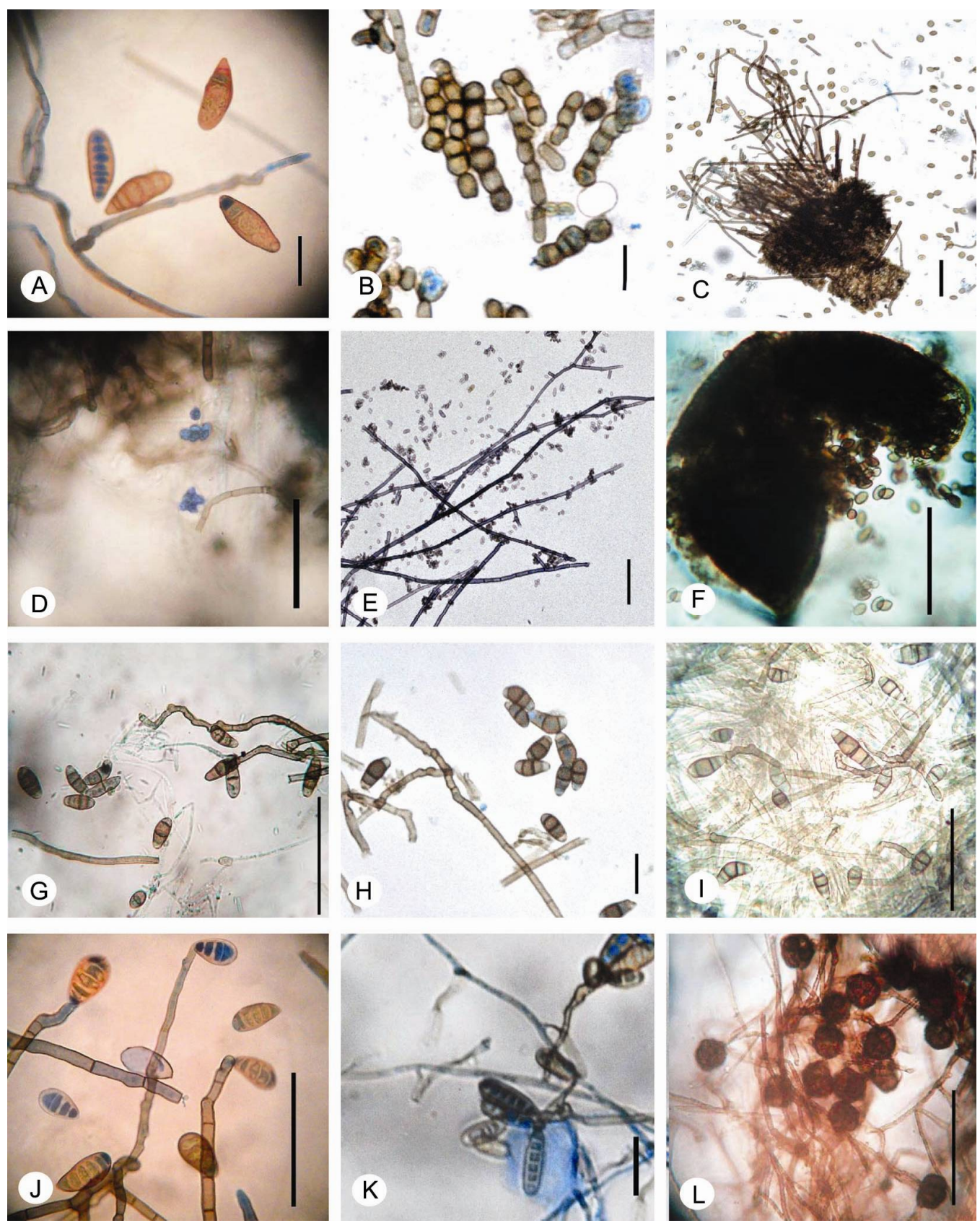

Fig. 2. Microphotograph showing conidiophores and conidia of A. Bipolaris victoriae, B. Bispora antenata C. Perithecium and ascospores of Chaetomium globosum, D. Conidia of Chaetophoma sp. E. Conidiophores and conidia of Cladosporium cladosporoides, F. Pycnidia and conidia of Conoithyium sp., conidiophores and conidia of G. Curvularia affinis, H. C. lunata, I. C. pallescens, J. Drechslera dematioidea, K. Drechslera hawaiiensis, L. Epicoccum purpurascens $(\mathrm{Bar}=50 \mu \mathrm{m})$. 
Specimen examined: BpLB infected leaves of wheat (Triticum aestivum L.), varietyBalaka, vill.-BARI, dist. - Gazipur, S Momtaz 62, 10 March 2010.

Coniothyrium Corda 1840. Icones fungorum hucusque cognitorum 4:38

(Fig. 2F)

Colonies greish black, cottony. Reverse grayish black. Pycnidia globose, ostiolate, 62.4-84 × 58.8-84 $\mathrm{mm}$ conidiophores reduced, simple, conidia small, ellipsoid, dark,1celled, $13.5 \times 6.3-8.1 \mu \mathrm{m}$.

Specimen examined: BpLB infected leaves of wheat (Triticum aestivum L.), varietyAnanda, vill.-WRC, dist. - Dinajpur, S Momtaz 138, 23 February, 2011.

Curvularia affinis Boedijn 1933. Bull. Jard. Bot. Buitenz. 13(1):130

(Fig. 2G)

Colonies cottony, effuse, grayish black. Conidiophores solitary, unbranched, straight. Conidia, pale brown, curved, 25-39.7 ×9.1-12.8 $\mu \mathrm{m}$.

Specimen examined: BpLB infected leaves of wheat (Triticum aestivum L.), varietyBarkat, vill.-BARI, dist.- Gazipur, S Momtaz 95, 10 February 2010.

Curvularia lunata (Wakker) Boedijn 1933. Bull. Jard. Bot. Buitenz. 13(1): 123

(Fig. 2H)

Colonies effuse grayish black or black, hairy, cottony or velvety. Conidiophores solitary, unbranched, straight or slightly undulating, geniculate, mid brown, septate, 37 $64 \times 9.2$ - 14.4. Conidia dark brown, mostly 3-septate, mostly curved, third cell from the base is broader and darker than others, broader cells mid brown, other cells paler, smooth, 22.5 - $31.2 \times 9.3-12.6 \mu \mathrm{m}$.

Specimen examined: BpLB infected leaves of wheat (Triticum aestivum L.), varietyProtiva, vill.-BARI, dist. - Gazipur, S Momtaz 69, 10 February 2010.

Curoularia pallescens Boedijn 1933. Bull. Jard. Bot. Buitenz. 13(1): 123

(Fig. 2I)

Colony effuse, gray, becoming black at maturity. Conidiophores solitary, mostly unbranched, straight, broader at the apex, up to $90.2 \mu \mathrm{m}$ long and 4.9 - 7 - $5 \mu \mathrm{m}$ thick. Conidia mostly 4-septate, slightly curved, smooth, 18.8 - $28 \times 7.8$ - $9.2 \mu \mathrm{m}$.

Specimen examined: BpLB infected leaves of wheat (Triticum aestivum L.), variety-Akber, vill.-BARI, dist.- Gazipur, S Momtaz 94, 10 February 2010.

Drechslera dematioidea (Bub. \& Wrob.) Subram. \& Jain 1966. Curr. Sci. 35: 354 (Fig. 2J)

On PDA medium colony blackish ash to black, reverse black, mycelium fluffy. Conidiophores light brown, short, slender, arising singly or in small group. Conidia yellowish brown, cylindrical to clavate, broader at the tip, tapering towards the base, ending in a wide dark scar, basal cell lighter in colour, thick walled, 2 - 7 pseudoseptate, $27.9-40.6 \times 14.1-16 \mu \mathrm{m}$.

Specimen examined: BpLB infected leaves of Wheat (Triticum aestivum L.), varietySaurav, vill.-Doripara, dist. - Joypurhat, S Momtaz 410, 4 April 2013. 
Drechslera hawaiiensis (Bugnicourt) ex M.B. Ellis; Subram. \& Jain 1966. Curr. Sci. 35: 354

(Fig. 2K)

Colony effuse, grayish black, reverse black. Conidiophores are solitary and brown in colour. Conidia pale to mid brown, slender, borne in clusters towards the apex, oblong or cylindrical, rounded at the ends, 4-7 (5) septate, 16.2 - 28.9 $\times 6.3-9 \mu \mathrm{m}$.

Specimen examined: BpLB infected leaves of Wheat (Triticum aestivum L.), variety-Seri82, vill.-BARI, dist. - Gazipur, S Momtaz 32, 3 April 2010.

Epicoccum purpurascens (E. nigrum Link) Ehrenb. ex Schlecht. 1824. Synop. Pl. crypt. :136

(Fig. 2L)

Colonies olivaceous green. Sporodochia globose, dark, up to $2 \mathrm{~mm}$. Conidiophores very short, unbranched, straight or flexuous, colourless to pale brown, smooth, 5-15 × 3-6 $\mu \mathrm{m}$. Conidia solitary, subspherical or pyriform, dark golden brown, often with a pale protuberant basal stalk cell, most commonly 15 - $25 \mu \mathrm{m}$. But smaller and much larger (up to $50 \mu \mathrm{m}$ ) conidia are formed sometimes.

Specimen examined: BpLB infected leaves of Wheat (Triticum aestivum L.), varietyGaurab, vill. - WRC, dist. - Dinajpur, S Momtaz 180, 24 March 2011.

Eurotium sp. 1809. Magazin der Gsellschaft Naturforschenden Freunde Berlin 3(1): 31

(Fig. 3A)

Colonies on PDA medium light yellowish, cottony, reverse orange red. Hyphae pale to mid brown, smooth, septate, branched, 1 - $4 \mu \mathrm{m}$ diameters. Cleistothecia superficial, yellow to light brown, globose to subglobose, 93.6 - $146.4 \mu \mathrm{m}$, asci 8 spored, 9.2 - $10.8 \mu \mathrm{m}$, ascospores $4.4-5.6 \times 5.2-6 \mu \mathrm{m}$.

Specimen examined: BpLB infected leaves of wheat (Triticum aestivum L.), varietyKanchan, vill.-BARI, dist.-Gazipur, S Momtaz 114, 13 March, 2011.

Fusarium moniliforme Sheldon 1904. Rep. Neb. Agric. Exp. Stn 17: 23-32

(Fig. 3B)

Mycelium extensive and cottony, white, often with some tinge of pink. Reverse pinkish yellow. Mycelia hyaline, profusely branched, septate. Conidiophores hyaline 0-2 septate. Phialides hyaline, $16-20 \times 3-4 \mu \mathrm{m}$. Conidia hyaline, variable, principally of two kinds. Microconidia 1-celled, ovoid or oblong, borne singly or in chains, $5-15 \times 2-3 \mu \mathrm{m}$. Macroconidia several-celled, slightly curved or bent at the pointed ends, 3 - 4 septate, conidia $25-35 \times 3-4 \mu \mathrm{m}, 5-6$ septate conidia $30-50 \times 3-5 \mu \mathrm{m}$.

Specimen examined: BpLB infected leaves of Wheat (Triticum aestivum L.), varietyKheri, vill.- BARI, dist. - Gazipur, S Momtaz 01, 10 March, 2010. 
Fusarium nivale (Fr.) Ces. 1839. Rabenh. Herb. Myc. Ed. 1, No. 1439.

(Fig. 3C)

Colonies white to pale peach to apricot. Mycelium sparse to densely floccose or felted, individual hyphae irregular. Conidia hyaline, variable, principally of two kinds, curved, broadly falcate with a pointed apex and flattened, wedge shape base, 3-septate, $14.3-28.4 \times 2.4-4.4$, macroconidia several-celled, slightly curved or bent at the pointed ends, typically canoe-shaped, microconidia 1-celled, ovoid or oblong, borne singly or in chains, some conidia intermediate, 2 or 3 celled, oblong or slightly curved, parasitic or saprophytic.

Specimen examined: Isolated from BpLB infected leaves of wheat (Triticum aestivum $\mathrm{L}$.), variety-Seri-82, vill.-BARI, dist. - Gazipur, S Momtaz 32, 3 April 2010.

Fusarium semitectum Berk. \& Rav. In Berkeley, Gravillea 3: 98, 1875.

(Fig. 3D)

Cultures at first white peach tinge and peach couloured from below. Macroconidia formed in aerial mycelium from loosely branched conidiophores. Macroconidia varied from 3 to 5 septate, curved with a wedge-shaped but not pedicellate basal cell and pointed apex. 3 septate conidia 19.8 - $26.8 \times 2.7-3.6 \mu \mathrm{m}$ and 5 septate conidia $22-40 \times 3.7$ - $4 \mu \mathrm{m}$. Microconidia aseptate pyriform to obovate, $10-12 \times 2.5-3.5 \mu \mathrm{m}$.

Specimen examined: BpLB infected leaves of wheat (Triticum aestivum L.), variety-Inia66, vill.-BARI, dist. - Gazipur, S Momtaz 55, 20 March 2011.

Nigrospora oryzae (Berk. \& Broome) Petch. 1924. J. Indian Bot. Soc.: 24

(Fig. 3E)

Colonies at first white, effuse with small shining black conidia, later brown or black when sporulation abundant. Mycelium immersed. Conidiophores solitary, ampulliform or subspherical, colorless. Conidia solitary, simple, spherical or broadly ellipsoidal, compressed dorsiventrally, black, shining, smooth, 0-septate. Conidiophores 3 - $7 \mu \mathrm{m}$ thick. Conidiogenous cells 6 - $9 \mu \mathrm{m}$ diam. Conidia 10 - 17 (mostly 14 - $15 \mu \mathrm{m}$ ) diam.

Specimen examined: BpLB infected leaves of wheat (Triticum aestivum L.), variety-, vill.WRC, dist. - Dinajpur, S Momtaz 112, 24 March 2011.

Nigrospora sacchari (Speg.) Mason 1927. Trans. Br. Mycol. Soc., 12(2-3): 152-165. (Fig. 3F)

Colonies at first white with small shining black conidia, later brown or black, sporulation abundant. Mycelium partly superficial. Conidiophores ampulliform or subspherical, colorless. Conidia solitary, simple, spherical or broadly ellipsoidal, compressed dorsiventrally, black, shining, smooth, 0-septate.

Conidiophores 4 - $7 \mu \mathrm{m}$ thick. Conidiogenous cells 7 - $9 \mu \mathrm{m}$ diam. Conidia 17-24 (mostly 20 - 22) $\mu \mathrm{m}$ diam.

Specimen examined: BpLB infected leaves of wheat (Triticum aestivum L.), varietyAnanda, vill.-WRC, dist. - Dinajpur, S Momtaz 140, 24 March 2011. 

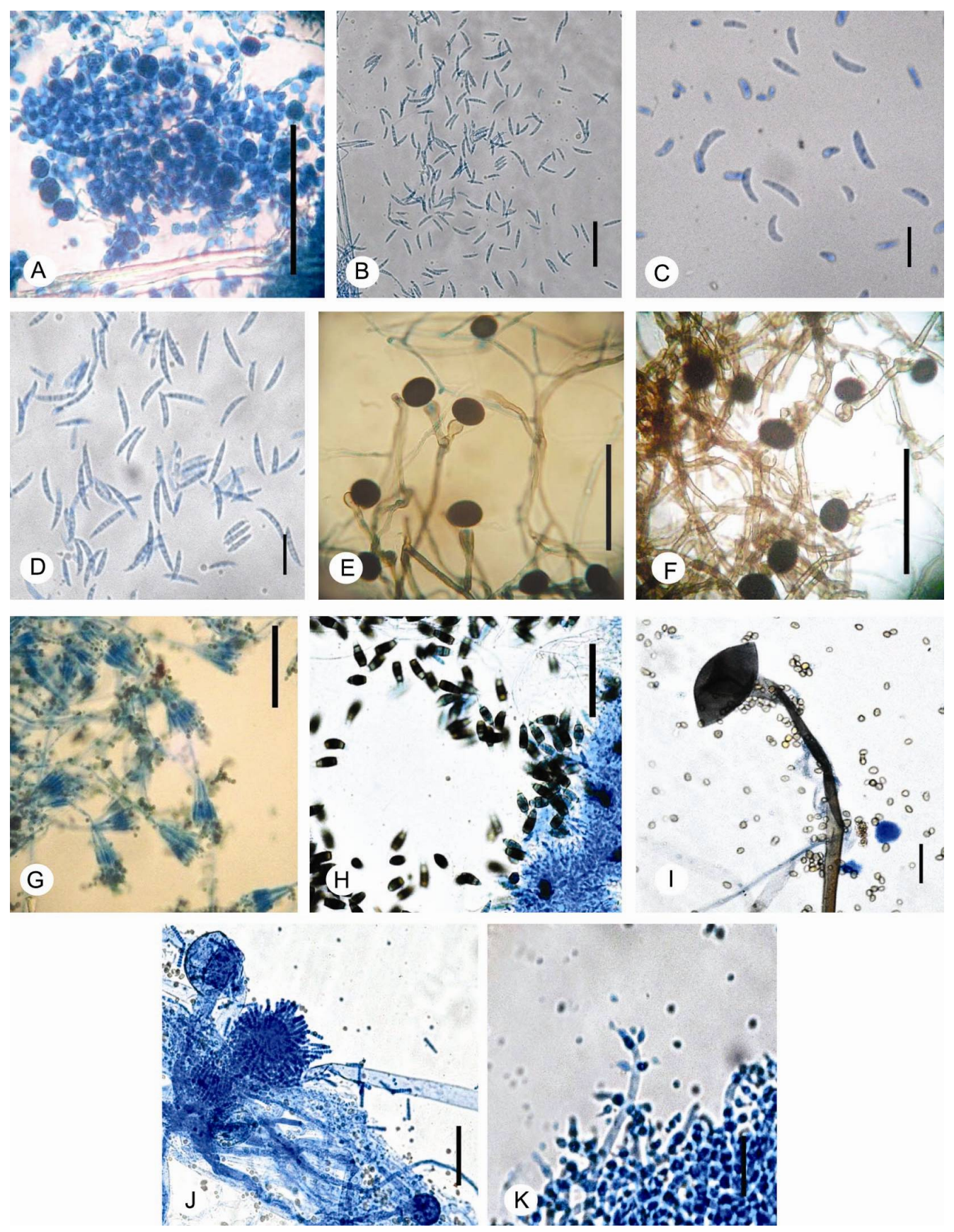

Fig. 3. A. Asci and ascospores of Eurotium sp., conidiophores and conidia of C. Fusarium moniliforme, D. F. nivale, E. F. semitectum, F. Nigrospora oryzae, G. N. sacchari, H. Penicillium digitatum, I. Pestalotiopsis guepinii, J. Rhizopus stolonifer; K. Syncephalastrum racemosum. and L. Trichoderma viride $(\mathrm{Bar}=50 \mu \mathrm{m})$. 
Penicillium digitatum (Fr.) Sacc. 1910. Bur. Anim. Ind., Bul. 118: 31-33

(Fig. 3G)

Colony small, cottony, greenish, reverse creamy. Hyphae creeping, septate, branched, hyaline. Conidiophores erect, apically irregularly verticillate-penicillately branched, $15-28 \times 3.5-5.0 \mu \mathrm{m}$. Conidia catenulate, spherical or elliptical, smooth, white in mass, commonly $3.5-5.0 \mu \mathrm{m}$.

Specimen examined: BpLB infected leaves of wheat (Triticum aestivum L.), varietyKalyansona, vill.-BARI, dist. - Gazipur, S Momtaz 17, 10 March 2010.

Pestalotiopsis guepinii (Desm.) Stay. Bulletin du Jardin Botanique de IEtat a Bruxelles 19(3): 312

(Fig. 3H)

Colonies white, cottony. Mycelia hyaline, septate, profusely branched, fruiting structure black, shining, conspicuous, condiomata $200 \mu \mathrm{m}$. Conidiophores short, hyaline, $10-15 \times 1-2 \mu \mathrm{m}$, mostly aseptate with 1-2 proliferation. Conidia blackish brown, mostly three septate with 2 - 5 hyaline appendages at the apex and short hyaline appendage at the base, apical appendages 16 - $33 \mu \mathrm{m}$ long and basal appendage $4-12 \mu \mathrm{m}$ long.

Specimen examined: BpLB infected leaves of wheat (Triticum aestivum L.), varietyBalaka, vill.-WRC, dist. - Dinajpur, S Momtaz 130, 24 March 2011.

Rhizopus stolonifer (Ehrenb.: Fr.) Vuillemin 1902. Toney Bot. Clup. 69: 592 - 616. (Fig. 3I)

Colonies grayish, fluffy. Reverse light gray. Mycelium coenocytic, well developed, branched and fluffy. Mycelium produces long conidiophores, 300-700 $\mu \mathrm{m}$ long and 3-4 $\mu \mathrm{m}$ in width, many aerial stolons also develop rhizoids at certain points. Directly above the rhizoids one or more sporangiospores are produced. The top of each sporangiophores becomes swollen as the latter reaches maturity and a sporangium is developed. Columella present. Sporangium produces non motile, brownish sporangiospores, 4 - 6 $\mu \mathrm{m}$ in diameter.

Specimen examined: BpLB infected leaves of wheat (Triticum aestivum L.), varietyShatabdi, vill.- Farmpara, dist. - Chuadanga, S Momtaz 324, 13 March 2013.

Syncephalastrum racemosum Cohn ex J. Schröt. 1886. Kryptogamen-Flora von Schlesien 3-1(2): 217

(Fig. 3J)

Colonies transparent, fluffy, grow very rapidly and fill the Petri dish with PDA medium 48 hours. Reverse is pale or yellowish-brown. Mycelium growing rapidly, abundantly branched. Sporangiophores are frequently branched and rather short. They end up in a vesicle ( $85 \mu \mathrm{m}$ in diameter). Around this vesicle are the merosporangia (4 - 6 $\times$ 9-60 $\mu \mathrm{m}$ ), which are filled with linear series (chains) of sporangiospores. Each merosporangium contains a single row of 3 - 18 merosporangiospores. Merosporangiospores ( $3-8 \mu \mathrm{m}$, may rarely reach $11 \mu \mathrm{m}$ in diameter) are one-celled and spherical to cylindrical in shape. 
Specimen examined: BpLB infected leaves of wheat (Triticum aestivum L.), varietyAghrani, vill.-BARI, dist. - Gazipur, S Momtaz 100, 13 February 2010.

Trichoderma viride Pers. 1794. Neues Magazin fur die Botanik 1: 92

(Fig. 3K)

Colony effuse, light green, hyphae elongate. Conidiophores hyaline, upright, much branched, bearing phialides single or in groups. Conidia hyaline, powdery mass, globose, 1-celled, ovoid, borne in small terminal clusters $3.5-5 \mu \mathrm{m}$, usually easily recognized by its rapid growth and green patches or cushions of conidia, saprophytic in soil or on wood, very common, some species reported as parasites on other fungi.

Specimen examined: Isolated from BpLB infected leaves of wheat (Triticum aestivum L.), variety-Kanchan, vill.- BARI, dist.- Gazipur, S Momtaz 324, 3 March 2012.

\section{Acknowledgement}

The first author (MSM) gratefully acknowledges the financial support by the Ministry of Science and Technology, Government of the People's Republic of Bangladesh through NST fellowship.

\section{References}

1. BBS 2017. Statistical Year Book of Bangladesh. Bangladesh Bureau of Statistics. Ministry of Planning, Government of Bangladesh.

2. Talukdar MJ 1974. Plant diseases in Bangladesh. Bangladesh J. Agril. Res. 1(1): 61-86.

3. Ahmed HU 1986. Prevailing Wheat Diseases in Bangladesh. In: Anonymous (ed.), Third National Wheat Training Workshop, Wheat Research Centre, BARI, Joydebpur, Gazipur. pp. 124-134.

4. Sadat MA and J Choi 2016. Wheat Blast: A New Fungal Inhabitant to Bangladesh Threatening World Wheat Production. Bangladesh J. Pl. Pathol. 33(2): 103-108.

5. Ahmed SM and CA Meisner 1996. Wheat research and development of Bangladesh, CIMMYTBangladesh. pp. 85.

6. Zillinsky FJ 1983. Common Diseases of Small Grain Cereals: A Guide to Identification. Mexico DF, Mexico. CIMMYT. pp.141.

7. Wiese MV 1998. Compendium of Wheat Diseases. (3rd Ed). APS Press, St. Paul Minnesota, USA. pp. 112.

8. Saari EE 1998. Leaf blight diseases and associated soil borne fungal pathogens of wheat in south and southeast Asia. In: Helminthosporium Blight of Wheat: Spot Blotch and Tan Spot. E Duveiller, HJ Dubin, J. Reeves and A McNab (Eds), Mexico DF, Mexico. CIMMYT. pp 37- 51.

9. Alam KB, PK Malaker, MA Shaheed, MU Ahmed, F Ahmed and MS Haque 1995. Yield loss assessment of wheat due to Bipolaris leaf blight in Bangladesh. Bangladesh J. Pl. Pathol. 11(1\&2): 35-38. 
10. Singh RV, AK Singh and SP Singh 1997. Distribution of pathogens causing foliar blight of wheat in India and neighbouring countries. In: Helminthosporium Blight of Wheat: Spot blotch and Tan Spot E. Duveiller, H.J. Dubin, J. Reeves and A. McNab (Eds.). 9-14 February, Mexico DF, Mexico. CIMMYT. pp. 59-62.

11. CAB (Commonwealth Agricultural Bureau) 1968. Plant Pathologist's Pocket Book. $1^{\text {st }}$ edition. The Commonwealth Mycological Institute, England. pp. 267.

12. Barnett HL and Hunter BB 2000. Illustrated genera of imperfect fungi. $4^{\text {th }}$ edition.Pub. Co. The American Phytopathological Society, St. Paul, Minnesota. pp. 185.

13. Booth C 1971. The Genus Fusarium. Commonwealth Mycological Institute, Kew, Surry, England. pp. 273.

14. Ellis MB 1971. Dematiaceous Hyphomycetes. Commonwealth Mycological Institute, England. pp. 608.

15. Ellis MB 1976. More Dematiaceous Hyphomycetes. Commonwealth Mycological Institute, England. pp. 608.

16. Ellis MB and JP Ellis 1997. Micro Fungi on Landplants. An Identification Handbook. The Commonwealth Mycological Institute, England. pp. 868.

17. Chidambaram P, SB Mathur and P Neergaard 1973. Identification of seed-borne Drechslera species. Friesia 10 (3): 165-207.

18. Sutton BC 1980. The Coemycetes, Fungi Imperfecti with Pycnidia, Acervuli and Stroma. Commonwealth Mycological Institute, Kew, Surrey, England. pp. 696. 